\title{
Immunohistochemical Expression of Antioxidant Enzyme PRDX6 in Oral Squamous Cell Carcinoma In Relation to Clinicopathological Parameters

\author{
Mustafa Gheni Taher $(\mathrm{MSc})^{1}$, Wasan Hamdi Younis $(\mathrm{PhD})^{2}$ and Muna Salih \\ $\operatorname{Merza}(\mathrm{PhD})^{3}$
}

\begin{abstract}
Background:The PRDX6 antioxidant family is important in the protection of cells against oxidants in humans. It is encoded by the PRDX6 gene. Peroxiredoxin 6 antioxidant enzyme (PRDX 6) has been reported in several cancers. However, the function of PRDX 6 in oral squamous cell carcinoma (OSCC), is far from being understood.

Objective:To normal corresponding oral mucosa in relation to clinicopathological parameters.

Patients and Methods: This study included 40 formalin-fixed, paraffin-embedded tissue blocks of primary OSCC cases and fifteen normal oral mucosa samples. Demographic data, clinical staging and histopathological grading were obtained. Immunohistochemically staining anti PRDX 6 antibody was done to illustrate its subcellular localization in normal and malignant cells.

Results: Females were affected more than males and the tongue was the most common site involved. PRDX 6 expression was high in OSCC than normal $(\mathrm{p}=0.001)$ with mean $\pm S d$ (60 \pm 18.8$)(23 \pm 9.8)$. Significant correlations were found with tumor size, lymph node and tumor stage ( $\mathrm{p}=0.008, \mathrm{p}=0.012, \mathrm{p}=0.011$ respectively) . Whereas no relation was observed concerning age, gender and tumor site.

Conclusion: Significant correlation of PRXD 6 anti oxidant enzyme expression with tumor size, lymph node involvement and clinical staging, suggesting its role in oral squamous cell carcinoma progression.
\end{abstract}

Key words: Oxidative stress, PRDC 6, squamous cell carcinoma.

Corresponding Author: Mostafa.ghany@yahoo.com

Received: $26^{\text {th }}$ October 2017

Accepted: $14^{\text {th }}$ November 2017

https://doi.org/10.26505/DJM

${ }^{1}$ Department of Pathology -College of Medicine- Diyala University - Diyala-Iraq.

${ }^{2}$ Department of Oral Pathology - College of Dentistry - Baghdad University- Baghdad - Iraq.

${ }^{3}$ Dentistry College - National University of Science and Technology - Nasiriya - Iraq.

\section{Introduction}

Regarding malignant head and neck tumors, squamous cell carcinoma (OSCC)of the head and neck is the most common epithelial neoplasia. It is the sixth most common cancer with an annual incidence of 500,000 and represent about $90 \%$ of all oral malignancies (Salian et al., 2016). The

survival of OSCC patients has not significantly improved in spite of development of many biological markers (Molinolo et al., 2009). Clinicopathological criteria are important for the management and prediction of outcome of patients. However, within a group of patients sharing 
the same features, there is still significant variation in prognosis (Thomas et al., 2005).

Oxidative stress is a serious imbalance between oxidation and antioxidants, however, it builds on definitions about oxidation, antioxidants, and balance (Sies, 2015). The definition of oxidation seems also simple: loss of electrons by a species, gain of oxygen, or loss of hydrogen. However, if something is oxidized, something else must be reduced. In biology, there is a pecking order of oxidants.

An antioxidant is any substance that, when present at low concentrations as compared with those of an oxidizable substrate, significantly delays or prevents the oxidation of that substrate (Azzi et al., 2004; Halliwell, 2004;Levonen et al., 2014). The chemical term an antioxidant is clearly different from a reducing agent. A reducing agent may even be a prooxidant if it reduces oxygen to free radicals or converts transition metal ions to lower oxidation states that react more readily with peroxides. Many biological reducing agents can be anti- or prooxidants, depending on the levels of $\mathrm{O} 2$ and transition metal ions around (Sies, 2015).

The Peroxiredoxin (PRDX) antioxidant protein family is preseny in a high range of species and protect cells against oxidants, in DNA is encoded by the PRDX6 gene. It is a part of the peroxiredoxin family of antioxidant enzymes (Cong et al., 2013).

The crystal structures of six PRDXs in mammals have been published in 1998, including four distinctive 2-Cys PRDXs (PRDX1, PRDX1I, TryP and AhpC), one atypical 2-Cys PRDX (PRDX V) and one 1Cys PRDX (PRDX6). PRDX2, 3, 5 and 6 planes were shown to be increased in malignant mesothelioma, in other hand PRDX2 and 3 were overexpressed in breast malignancy specimens compared with no malignant tissues (Kinnula et al., 2002; Lee et al., 2015).

The PRDXs are antioxidants, so they support survival and tumor maintenance by covering cells from oxidative stress-induced apoptosis (Neumann et al., 2007). In a study on ovarian cancer, high expression of PRDX 6 attenuates cisplatin- act to apoptosis in human ovarian malignancy cells (Pak et al., 2011). In other side, reduction of PRDX6 expression increased peroxide-induced cell death in liver cancer cells (Walsh et al., 2009). The invasion and metastasis promoting actions of PRDX6 has been found in lung cancer cells through activation of Akt via activation of phosphoinositiede 3-kinase (PI3K) and p38 kinase (Lee et al., 2009; Ho et al., 2010). The activity of PRDX6 contributes to the metastatic ability of lung cancer cells by stimulating invasion components including PI3K, Akt, and uPA (Ho et al., 2010). It was also reported that PRDX6 expression in lung cancer cells was significantly associated with tumor progression (Schremmer et al., 2007).

Jhang et al., in 2010 approve that targeting PRDX6 may sensitize cancer cells to ROSproducing therapeutic treatments, such as anticancer drugs and radiation (Jhang et al., 2011). In 2013, Rolfs reported that, the potential usefulness of PRDX6 activators or 
inhibitors for controlling different stages of skin carcinogenesis (Rolfs et al., 2013). Other recent research showed that peroxiredoxin 6 can be regarded as a prophylactic radioprotective agent (Sharapov et al., 2016). The immune marker AntiPeroxiredoxin 6 antibody is Rabbit monoclonal to Peroxiredoxin 6, belongs to the ahpC/TSA family. Rehydrin subfamily. Contains 1 thioredoxin domain and which have Predicted molecular weight $25 \mathrm{kDa}$.

The present study investigated the expression levels of PRDX 6 in OSCC in comparison to normal oral mucosa, which have previously been found to be highly expressed in various malignant neoplasms. Thus, the valuable predictive factors of OSCC are required to prevent the tumorigenesis, progression and recurrence of the disease.

\section{Patients and Methods}

Fifty-five formalin-fixed, paraffinembedded tissue blocks, including fifteen normal oral mucosa (alveolar mucosa obtained during orthodontic extractions or implantation process). In addition, forty primary squamous cell carcinoma surgical specimens obtained from the achieves of department of oral pathology / college of Dentistry- Baghdad University, and some private laboratories were included in this study. Data concerning patient's age, gender, site, clinical staging and histopathological grading were obtained from the associated patients' reports. Tumor histopathological sections were reviewed by two pathologists, and the representative paraffin blocks were selected. A section of 4- $\mu \mathrm{m}$ thickness was cut from each tissue block and mounted on positively charged slides (Esco, USA) to be stained with monoclonal antibodies to PRDX6 antioxidant marker (Abcam ab133348 UK). Negative and positive tissue controls were included into each immunohistochemical run.

The slides were baked in hot air oven at $65^{\circ} \mathrm{C}$ overnight. Sections were sequentially de waxed through a series of xylene, graded alcohol and water immersion steps. Drops of hydrogen peroxide block were added to slides were in a ready to use package (ab126738). All slides were followed by the application of the primary antibodies with a dilution of 1:250. The slides were incubated for $1 \mathrm{~h}$ at $37^{\circ} \mathrm{C}$ and then kept at $4^{\circ} \mathrm{C}$ in a humid chamber overnight. Next day, after washing with PBS (Phosphate Bupher Solution), biotinylated antimouse IgG were applied to the sections, incubated and rinsed with a stream of PBS. Conjugated antibodies were visualized with $\mathrm{DAB}$ chromogen. Sections were counterstained with Mayer's hematoxylin for 1-2 min, dehydrated and mounted, all these laboratory procedures were performed in the college of dentistry/ Laboratory of department of oral pathology.

Estimation of the immunohistochemical results: The immunostaining results were blindly understood by two self-regulating oral pathologists under the light microscope at a magnification of 400. A semi quantitative scoring scheme was used to determine the percentage of positively stained cells as brown cytoplasmic 
expression. The ratio of positive immunostained cells was graded and scored as follows: $0=$ no stained cells; $1=1-25 \%$ positive cells; $2=26-50 \%$ positive cells; $3=$ $51-75 \%$ positive cells; and $4=$ extra than $75 \%$ positive cells. (Huang et al., 2011).

\section{Statistical Analysis}

Statistical analyses were performed utilizing computer assisted SPSS version (12) (SPSS professional 2005). The parametric statistical tests were used namely two -tailed T-test and ANOVAs table. The nonparametric statistical tests were used namely Chi- square test. P-values less than (0.05) level of significance was considered statistically significant at $95 \%$ confidence. The statistical correlations were measured by Pearson's and Spearman's linear correlation coefficient.

\section{Results}

As shown in table (1), males were slightly more frequent among healthy controls group, $9(60 \%)$. The age of healthy controls ranged between 25 to 68 years of age with a mean of $49+/-11$ years of age. The highest category of controls were $40-59$ years of age $(66.7 \%)$. Regarding OSCC sample patients, females were slightly more frequent among cases group $29(72.5 \%)$. The age of cases group ranged between 19 - 81 years of age with a mean of $54+/-15$ years of age. In addition, the highest category of this study group were $40-59$ years of age $(42.5 \%)$, as shown in table (2). According to site distribution, the tongue was the most frequently reported site of tumor $16(40.0 \%)$ figure (1).
When clinical staging of the studied sample was evaluated, the tumor size was less than 2 $\mathrm{cm}$ in $14(35 \%)$ of cases, while it was exceptionally large $(>4 \mathrm{~cm})$ in $11(27.5 \%)$ of cases and invasive into adjacent area in $2(5 \%)$ of cases only. No lymph node involvement was observed in 19(47.5\%) of cases, while it reached to the stage of $\mathrm{N} 2$ (single ipsilateral lymph node involvement 3 to $6 \mathrm{~cm})$ in $5(12.5 \%)$ of cases. In about a quarter of cases $11(27.5 \%)$ the tumor was at Stage-I, while it reached to stage-IV in $6(15 \%)$ of cases only. table (3). Well differentiated OSCC comprised more than half $22(55 \%)$ of the studied cases group Figure(2).

Peroxiredoxin 6 monoclonal antibody IHC expression subcellular localization was in the cytoplasm (Figures 3,4,5,6,7). The mean percentage of positively stained cells with peroxiredoxin 6 was significantly higher $(\mathrm{p}=$ 0.001) in OSCC group than healthy control group with mean $\pm \mathrm{Sd}(60 \pm 18.8)$ and $(23 \pm 9.8)$ respectively. The median score of peroxiredoxin 6 was significantly higher $(\mathrm{p}=$ 0.001) with (strong staining 51-74\%) in OSCC group than control group (weak staining 1-25\%) (Figure 8, Tables 4A,4B). Most of healthy controls were in group (4059) years of age and all of them showed weak expression (1-25\%) with a non significant statistical relations were found neither with age nor with gender (Tables 5A,5B).

A similar non significant statistical relations were found, between study group of OSCC neither with age, gende,r nor with site. Most 
OSCC cases were present in the tongue that showed strong expression and the less site was labial mucosa which showed weak expression (Tables 6A,6B,6C).

The median score category of peroxiredoxin 6 was lowest (moderate staining) among cases with $\mathrm{T} 1$ category (smallest tumor size $\leq$ $2 \mathrm{~cm}$ ) and increase with higher $\mathrm{T}$ stage to reach its highest median score of very strong among cases with highest $\mathrm{T}$ stage (tumor invading adjacent area). The observed difference in median score of peroxiredoxin 6 between tumor $\mathrm{T}$ staging was statistically significant. Moreover, this marker showed a statistically significant moderately strong positive linear correlation with tumor $\mathrm{T}$ stage ( $\mathrm{r}=0.543)$ (Table 7). In a similar context, PRDX6 had a significant relation with lymph node involvement, the observed difference in median score of peroxiredoxin 6 between tumor $\mathrm{N}$ staging was statistically significant. This marker showed a statistically significant moderately strong positive linear correlation with tumor $\mathrm{N}$ stage $(\mathrm{r}=0.474)$ (Table 8$)$. As shown in table 9 , the median score category of peroxiredoxin 6 was lowest (moderate staining) among cases with tumors in the early stage (Stage-I) and progressively increase in magnitude of marker score to reach its highest value (very strong staining category score) among OSCC cases with highest tumor stage (Stage-IV). The observed difference in median score of peroxiredoxin 6 among the four tumor stages was statistically significant. Also, this marker showed a statistically significant moderately strong positive linear correlation with tumor $\mathrm{N}$ stage $(\mathrm{r}=0.455)$.

The peroxiredoxin 6 was associated with a high validity in differentiating between OSCC cases and healthy controls, since its ROC area was high (0.94), which would provide an excellent test, diagnosing OSCC separating it from a healthy person (Table 10). As shown in table 12 , the optimum cutoff value for peroxiredoxin 6 score when used as a test to diagnose OSCC differentiating it from healthy controls is moderate or higher score, since it was associated with the highest accuracy (90.7\%). In addition, this cut-off value is also the one associated with highest sensitivity (95\%). The confidence in a positive diagnosis (real presence of OSCC) would increase to $97.7 \%$ in a clinical context where having the tumor is highly probable on other clinical evidence (pretest probability $=90 \%$ ). Raising the cutoff value to the strong score or higher provides a test marker of perfect specificity (100\%). Testing positive at this highly specific cut-off value (obtaining a marker score of strong or very strong category) would establish a possible diagnosis of OSCC with $100 \%$ confidence in any clinical situation. 
Immunohistochemical Expression of Antioxidant Enzyme PRDX6 in Oral Squamous Cell Carcinoma In Relation to Clinicopathological Parameters Mustafa Gheny Taher

Table (1): Frequency distribution of healthy controls group by selected variables.

\begin{tabular}{|c||l|c||c|}
\hline \hline & & N & \% \\
\hline 1. & Gender & & \\
\hline & Female & 6 & 40.0 \\
\hline \hline & Male & 9 & 60.0 \\
\hline \hline & Total & 15 & 100.0 \\
\hline \hline $\mathbf{2 .}$ & Age (years) & & \\
\hline \hline & $<40$ & 3 & 20.0 \\
\hline & $40-59$ & 10 & 66.7 \\
\hline \hline & $60+$ & 2 & 13.3 \\
\hline & Total & 15 & 100.0 \\
\hline
\end{tabular}

Table (2): Frequency distribution of cases group (OSCC) by selected variables.

\begin{tabular}{|c|l||c||c|}
\hline & & N & \% \\
\hline \hline 1. & Gender & & \\
\hline \hline & Female & 29 & 72.5 \\
\hline \hline & Male & 11 & 27.5 \\
\hline \hline & Total & 40 & 100.0 \\
\hline \hline $\mathbf{2 .}$ & Age (years) & & \\
\hline \hline & $<40$ & 7 & 17.5 \\
\hline \hline & $\cdots \cdots \cdots \cdot$ & 17 & 42.5 \\
\hline \hline & $60+$ & 16 & 40.0 \\
\hline \hline & Total & 40 & 100.0 \\
\hline \hline
\end{tabular}

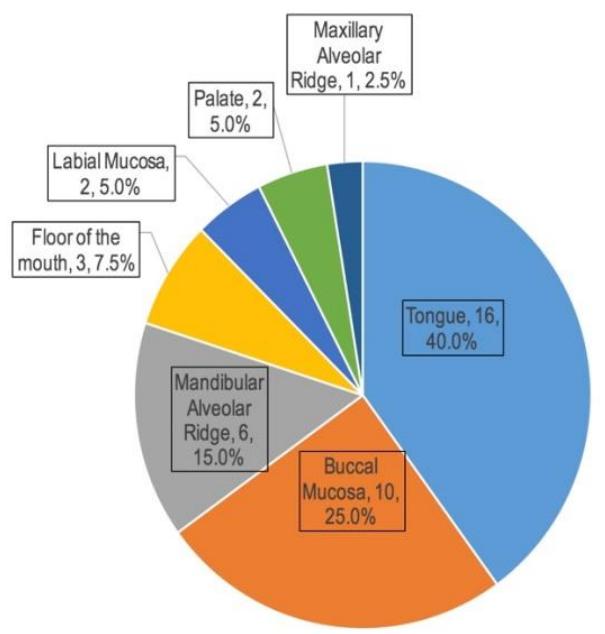

Figure (1): Pie chart showing the relative frequency of selected tumor locations $(\mathrm{N}=40)$. 
Immunohistochemical Expression of Antioxidant Enzyme PRDX6 in Oral Squamous Cell Carcinoma In Relation to Clinicopathological Parameters Mustafa Gheny Taher

Table (3): Frequency distribution of cases group (OSCC) by selected variables.

\begin{tabular}{|r|l|r||r|}
\hline & & $\mathbf{N}$ & \% \\
\hline \hline $\mathbf{1 .}$ & T (Tumor Size) & & \\
\hline \hline & $\leq 2 \mathrm{~cm}$ & 14 & 35.0 \\
\hline \hline & $2-4 \mathrm{~cm}$ & 13 & 32.5 \\
\hline \hline & Invasive to adjacent area & 11 & 27.5 \\
\hline & Total & 2 & 5.0 \\
\hline \hline $\mathbf{2 .}$ & N (lymph Node involvement) & & 100.0 \\
\hline \hline & No LN involvement & 19 & 47.5 \\
\hline \hline & N1 metastasis to single ipsilateral LN $\leq 3 \mathrm{~cm}$ & 16 & 40.0 \\
\hline \hline & N2 metastasis to single ipsilateral LN 3 - 6 cm & 5 & 12.5 \\
\hline \hline & Total & 40 & 100.0 \\
\hline \hline 3. & Tumor stage & & \\
\hline \hline & Stage I & 11 & 27.5 \\
\hline \hline & Stage II & 6 & 15.0 \\
\hline \hline & Stage III & 17 & 42.5 \\
\hline \hline & Stage IV & 6 & 15.0 \\
\hline \hline & Total & 40 & 100.0 \\
\hline
\end{tabular}

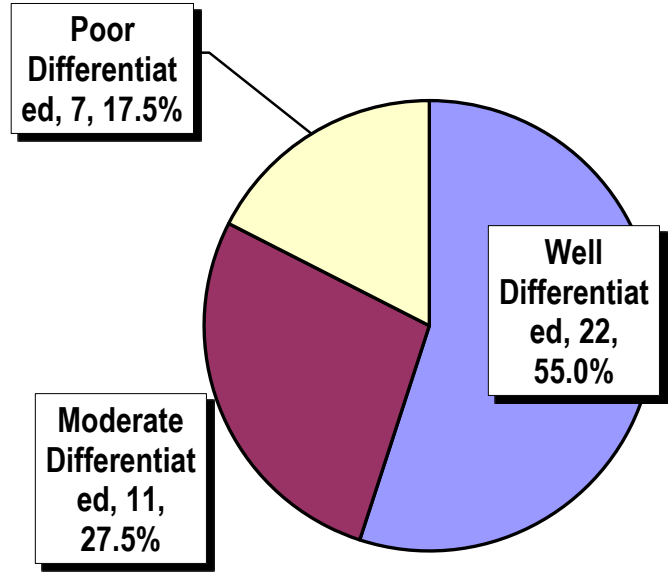

Figure (2): Pie chart showing the relative frequency of cases group by degree of tumor differentiation $(\mathrm{N}=40)$. 
Immunohistochemical Expression of Antioxidant Enzyme PRDX6 in Oral Squamous Cell Carcinoma In Relation to Clinicopathological Parameters Mustafa Gheny Taher

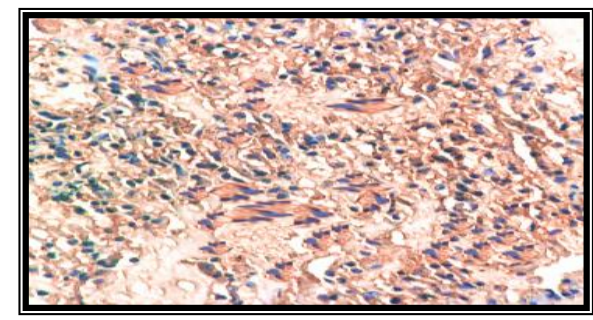

Figure (3):Photomicrograph showing Positive brown cytoplasmic expression of PRDX6 A immunostaining in Human brain (Positive Control) (Original magnification X400).

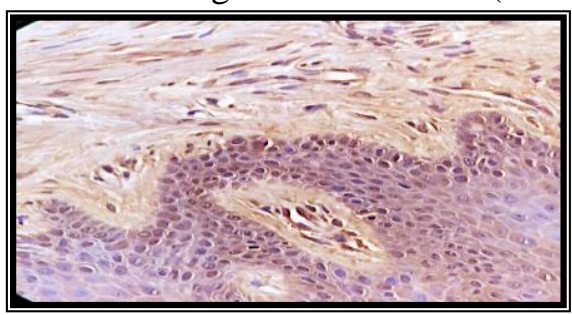

Figure(4):Photomicrograph showing Positive brown cytoplasmic expression of peroxiredoxin 6 in normal oral mucosa (Original magnification $\mathrm{X} 400)$.

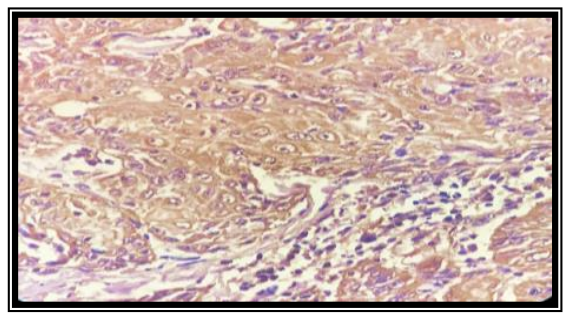

Figure(6):Photomicrograph showing Positive brown cytoplasmic expression of peroxiredoxin 6 in moderately differentiated OSCC (Original magnification X400).

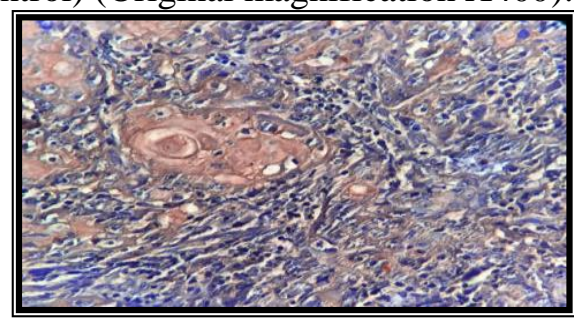

Figure(5):Photomicrograph showing Positive brown cytoplasmic expression of peroxiredoxin 6 in well differentiated OSCC (Original magnification).

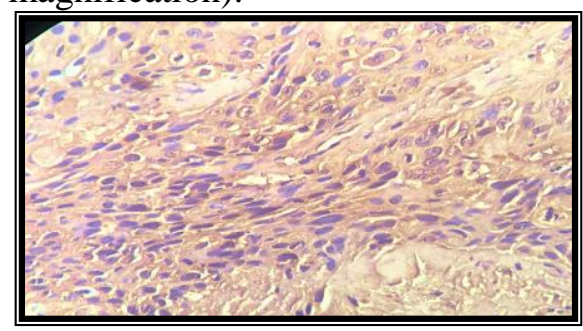

Figure(7):Photomicrograph showing Positive brown cytoplasmic expression of peroxiredoxin 6 in poorly differentiated OSCC (Original magnification X400).

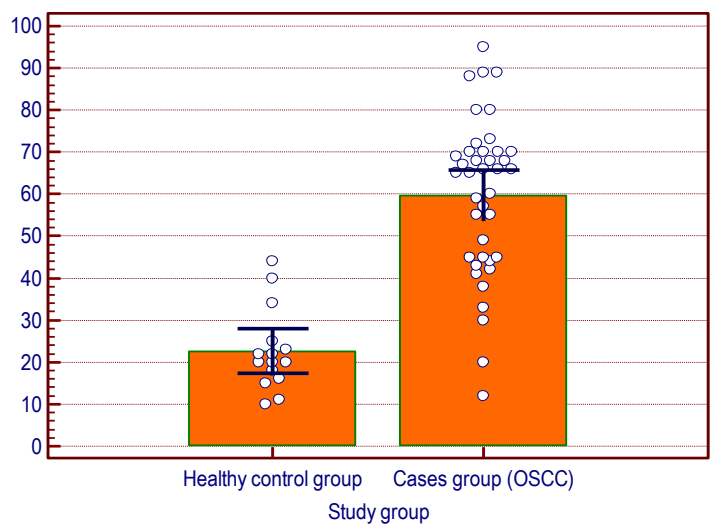

Figure (8): Dot diagram with error bars showing the case-control difference in mean (with its 95\% confidence interval) peroxiredoxin 6 percent positive cells. 
Immunohistochemical Expression of Antioxidant Enzyme PRDX6 in Oral Squamous Cell Carcinoma In Relation to Clinicopathological Parameters Mustafa Gheny Taher

Table (4A): Case-control difference in mean percentage of cells positive for selected markers.

\begin{tabular}{|c|c|c|c|}
\hline & \multicolumn{2}{|c|}{ Study group } & \\
\hline & Healthy control group & 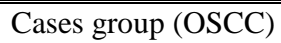 & $\mathrm{P}$ \\
\hline "peroxiredoxin 6-percent positive cell & & & $\angle 0.001$ \\
\hline Range & (10 to 44$)$ & (12 to 95$)$ & \\
\hline Mean & 23 & 60 & \\
\hline SD & 9.8 & 18.8 & \\
\hline$\overline{\mathrm{SE}}$ & 2.5 & 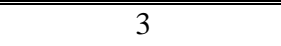 & \\
\hline $\mathrm{N}$ & 15 & 40 & \\
\hline
\end{tabular}

* NS Non-significant relation $(\mathrm{p}>0.0)$

Table (4B): Case-control difference in median value of selected marker scores.

\begin{tabular}{|c|c|c|c|c|c|}
\hline & \multicolumn{4}{|c|}{ Study group } & \\
\hline & \multicolumn{2}{|c|}{$\begin{array}{l}\text { Healthy control } \\
\text { group }\end{array}$} & \multicolumn{2}{|c|}{$\begin{array}{c}\text { Cases group } \\
\text { (OSCC) }\end{array}$} & \\
\hline & $\mathrm{N}$ & $\%$ & $\mathrm{~N}$ & $\%$ & $\overline{\mathrm{P}}$ \\
\hline peroxiredoxin 6 score & & & & & $<0.001$ \\
\hline Negative $(0 \%)$ & 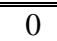 & 0.0 & 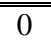 & 0.0 & \\
\hline Weak (1-25\%) & 12 & 80.0 & 2 & 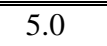 & \\
\hline Moderate (26-50\%) & 3 & 20.0 & 11 & 27.5 & \\
\hline Strong $(51-75 \%)$ & 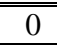 & 0.0 & 21 & 52.5 & \\
\hline "Very strong (76-100\%) & 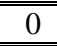 & 0.0 & 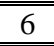 & 15.0 & \\
\hline Total & 15 & 100.0 & 40 & 100.0 & \\
\hline Median & \multicolumn{2}{|c|}{ Weak $(1-25 \%)$} & \multicolumn{2}{|c|}{ Strong (51-75\%) } & \\
\hline Mean Rank & \multicolumn{2}{|c|}{10.3} & \multicolumn{2}{|c|}{34.6} & \\
\hline
\end{tabular}

* NS Non-significant relation ( $>>0.05)$

Table (5A): The median value of selected marker scores by age group among healthy controls.

\begin{tabular}{|c|c|c|c|c|c|c|c|}
\hline & \multicolumn{6}{|c|}{ Age (years) } & \multirow[b]{3}{*}{$\mathrm{P}$} \\
\hline & \multicolumn{2}{|c|}{$<40$} & \multicolumn{2}{|c|}{ (40-59 } & \multicolumn{2}{|c|}{$60+$} & \\
\hline & $\overline{\mathrm{N}}$ & $\%$ & $\mathrm{~N}$ & $\%$ & $\mathrm{~N}$ & $\%$ & \\
\hline $\begin{array}{l}\text { peroxiredoxin 6- } \\
\text { score }\end{array}$ & & & & & & & $0.68[\mathrm{NS}]$ \\
\hline "Negative $(0 \%)$ & 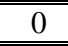 & 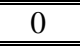 & 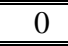 & 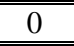 & 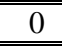 & 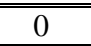 & \\
\hline Weak $(1-25 \%)$ & 2 & 66.7 & 8 & 80 & 2 & 100 & \\
\hline Moderate (26-50\%) & 1 & 33.3 & 2 & 20 & 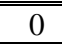 & 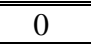 & \\
\hline Strong $(51-75 \%)$ & "0 & 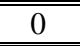 & 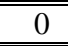 & 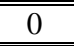 & 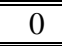 & 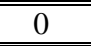 & \\
\hline $\begin{array}{l}\text { Very strong (76- } \\
100 \%)\end{array}$ & 0 & 0 & 0 & 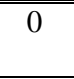 & 0 & 0 & \\
\hline Total & 3 & 100 & 10 & 100 & 2 & 100 & \\
\hline Median & \multicolumn{2}{|c|}{ Weak (1-25\%) } & \multicolumn{2}{|c|}{ Weak (1-25\%) } & \multicolumn{2}{|c|}{ Weak (1-25\%) } & \\
\hline "Mean Rank & \multicolumn{2}{|c|}{9} & \multicolumn{2}{|c|}{8} & \multicolumn{2}{|c|}{6.5} & \\
\hline $\mathrm{r}=-0.21 \mathrm{P}=0.45[\mathrm{NS}]$ & & & & & & & \\
\hline
\end{tabular}

* NS Non-significant relation ( $p>0.05$ ) 
Immunohistochemical Expression of Antioxidant Enzyme PRDX6 in Oral Squamous Cell Carcinoma In Relation to Clinicopathological Parameters

Mustafa Gheny Taher

Table (5B): The median value of selected marker scores by gender among healthy controls.

\begin{tabular}{|c|c|c|c|c|c|}
\hline & \multicolumn{4}{|c|}{ "Gender } & \\
\hline & \multicolumn{2}{|c|}{ Female } & \multicolumn{2}{|c|}{ Male } & \\
\hline & $\overline{\mathrm{N}}$ & $\%$ & $\bar{N}$ & $\%$ & $\bar{P}$ \\
\hline peroxiredoxin 6-score & & & & & $0.8[\mathrm{NS}]$ \\
\hline Negative $(0 \%)$ & 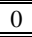 & 0 & 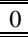 & 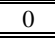 & \\
\hline Weak (1-25\%) & 5 & 83.3 & 7 & 77.8 & \\
\hline Moderate (26-50\%) & 1 & 16.7 & 2 & 22.2 & \\
\hline Strong $(51-75 \%)$ & 0 & 0 & 0 & 0 & \\
\hline "Very strong (76-100\%) & 0 & 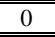 & 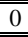 & 0 & \\
\hline Total & 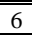 & 100 & 9 & 100 & \\
\hline Median & \multicolumn{2}{|c|}{ Weak (1-25\%) } & \multicolumn{2}{|c|}{ Weak (1-25\%) } & \\
\hline Mean Rank & \multicolumn{2}{|c|}{7.8} & \multicolumn{2}{|c|}{8.2} & \\
\hline
\end{tabular}

* NS Non-significant relation ( $\mathrm{p}>0.05)$

Table (6A): The median value of selected marker scores by age group among OSCC cases group.

\begin{tabular}{|c|c|c|c|c|c|c|c|}
\hline & \multicolumn{6}{|c|}{ Age (years) } & \\
\hline & \multicolumn{2}{|c|}{$<40$} & \multicolumn{2}{|c|}{$40-59$} & \multicolumn{2}{|c|}{$60+$} & \\
\hline & $\mathrm{N}$ & $\%$ & $\mathrm{~N}$ & $\%$ & $\mathrm{~N}$ & $\%$ & $\mathrm{P}$ \\
\hline peroxiredoxin 6-score & & & & & & & $0.44[\mathrm{NS}]$ \\
\hline Negative $(0 \%)$ & 0 & 0 & 0 & 0 & 0 & 0 & \\
\hline Weak (1-25\%) & 1 & 14.3 & 0 & 0 & 1 & 6.3 & \\
\hline Moderate (26-50\%) & 3 & 42.9 & 4 & 23.5 & 4 & 25 & \\
\hline Strong (51-75\%) & 2 & 28.6 & 11 & 64.7 & 8 & $\overline{50}$ & \\
\hline $\begin{array}{l}\text { Very strong (76- } \\
100 \%)\end{array}$ & 1 & 14.3 & 2 & 11.8 & 3 & 18.8 & \\
\hline Total & 7 & 100 & 17 & 100 & 16 & 100 & \\
\hline Median & \multicolumn{2}{|c|}{ Moderate $(26-50 \%)$} & \multicolumn{2}{|c|}{ Strong $(51-75 \%)$} & \multicolumn{2}{|c|}{ Strong $(51-75 \%)$} & \\
\hline Mean Rank & \multicolumn{2}{|c|}{15.9} & \multicolumn{2}{|c|}{21.8} & \multicolumn{2}{|c|}{21.1} & \\
\hline
\end{tabular}

Table (6B): The median value of selected marker scores by gender among OSCC cases group.

\begin{tabular}{|c|c|c|c|c|c|}
\hline & \multicolumn{4}{|c|}{ Gender } & \\
\hline & \multicolumn{2}{|c|}{ Female } & \multicolumn{2}{|c|}{ Male } & \\
\hline & $\mathrm{N}$ & $\%$ & $\mathrm{~N}$ & $\%$ & $\overline{\mathrm{P}}$ \\
\hline peroxiredoxin 6-score & & & & & $0.37[\mathrm{NS}]$ \\
\hline Negative $(0 \%)$ & 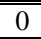 & 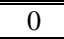 & 0 & 0 & \\
\hline Weak (1-25\%) & 1 & 3.4 & 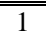 & 9.1 & \\
\hline Moderate (26-50\%) & 10 & 34.5 & 1 & 9.1 & \\
\hline Strong $(51-75 \%)$ & 14 & 48.3 & 7 & 63.6 & \\
\hline "Very strong (76-100\%) & 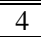 & 13.8 & 2 & 18.2 & \\
\hline Total & 29 & 100 & 11 & 100 & \\
\hline Median & \multicolumn{2}{|c|}{ Strong $(51-75 \%)$} & \multicolumn{2}{|c|}{ "Strong $(51-75 \%)$} & \\
\hline Mean Rank & \multicolumn{2}{|c|}{19.6} & \multicolumn{2}{|c|}{23} & \\
\hline
\end{tabular}

Table (6C): The median value of selected marker scores by site among OSCC cases.

\begin{tabular}{|c|c|c|c|c|c|c|c|c|c|c|c|c|c|c|c|}
\hline & \multicolumn{14}{|c|}{ Tumor site } & \\
\hline & \multicolumn{2}{|c|}{ Tongue } & \multicolumn{2}{|c|}{$\begin{array}{c}\text { Mandibular } \\
\text { Alveolar Ridge } \\
\end{array}$} & \multicolumn{2}{|c|}{$\begin{array}{c}\begin{array}{c}\text { Floor of the } \\
\text { mouth }\end{array} \\
\end{array}$} & \multicolumn{2}{|c|}{ Buccal Mucosa } & \multicolumn{2}{|c|}{ Labial Mucosa } & \multicolumn{2}{|c|}{$\begin{array}{c}\text { Maxillary } \\
\text { Alveolar Ridge } \\
\end{array}$} & \multicolumn{2}{|c|}{ Palate } & \\
\hline & $\mathrm{N}$ & $\%$ & $\mathrm{~N}$ & $\%$ & $\mathrm{~N}$ & $\%$ & $\mathrm{~N}$ & $\%$ & $\mathrm{~N}$ & $\%$ & $\mathrm{~N}$ & $\%$ & $\mathrm{~N}$ & $\%$ & $\mathrm{P}$ \\
\hline $\begin{array}{l}\text { peroxiredoxin 6-percent } \\
\text { positive cell-categories }\end{array}$ & & & & & & & & & & & & & & & $\begin{array}{c}0.32[\mathrm{~N} \\
\mathrm{S}] \\
\end{array}$ \\
\hline Weak (1-25\%) & 2 & $\begin{array}{l}11.8 \\
\end{array}$ & 3 & 33.3 & 1 & 20.0 & 2 & 18.2 & 1 & 33.3 & 3 & 60.0 & 2 & "40.0 & \\
\hline Moderate (26-50\%) & 4 & 23.5 & 1 & 11.1 & 1 & 20.0 & 4 & 36.4 & 0 & 0.0 & 2 & 40.0 & 2 & 40.0 & \\
\hline Strong (51-75\%) & 10 & 58.8 & 4 & 44.4 & 2 & 40.0 & 3 & 27.3 & 2 & 66.7 & 0 & 0.0 & 0 & 0.0 & \\
\hline Very strong (76-100\%) & 1 & 5.9 & 1 & 11.1 & 1 & 20.0 & 2 & 18.2 & 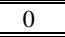 & 0.0 & 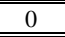 & 0.0 & 1 & 20.0 & \\
\hline Total & 17 & 100.0 & 9 & 100.0 & 5 & 100.0 & 11 & 100.0 & 3 & 100.0 & 5 & 100.0 & 5 & 100.0 & \\
\hline Median & \multicolumn{2}{|c|}{$\begin{array}{l}\text { Strong (51- } \\
75 \%) \\
\end{array}$} & \multicolumn{2}{|c|}{$\begin{array}{c}\text { Strong }(51- \\
75 \%) \\
\end{array}$} & \multicolumn{2}{|c|}{$\begin{array}{c}\text { Strong }(51- \\
75 \%) \\
\end{array}$} & \multicolumn{2}{|c|}{$\begin{array}{l}\text { Moderate (26- } \\
50 \%) \\
\end{array}$} & \multicolumn{2}{|c|}{$\begin{array}{c}\text { Strong }(51- \\
75 \%) \\
\end{array}$} & \multicolumn{2}{|c|}{ Weak $(1-25 \%)$} & \multicolumn{2}{|c|}{$\begin{array}{c}\text { Moderate (26- } \\
50 \%) \\
\end{array}$} & \\
\hline Mean rank & 32 & & 28.1 & & 31.9 & & 29.4 & & 28.5 & & 13.1 & & 22.1 & & \\
\hline
\end{tabular}

* NS Non-significant relation $(\mathrm{p}>0.05)$ 
Immunohistochemical Expression of Antioxidant Enzyme PRDX6 in Oral Squamous Cell Carcinoma In Relation to Clinicopathological Parameters

Mustafa Gheny Taher

Table (7): The median value of selected marker scores by tumour size among OSCC cases group.

\begin{tabular}{|c|c|c|c|c|c|c|c|c|c|}
\hline & \multicolumn{8}{|c|}{$\begin{array}{l}\text { T (Tumor Size) } \\
\end{array}$} & \\
\hline & \multicolumn{2}{|c|}{$\leq 2 \mathrm{~cm}$} & \multicolumn{2}{|c|}{$2-4 \mathrm{~cm}$} & \multicolumn{2}{|c|}{$>4 \mathrm{~cm}$} & \multicolumn{2}{|c|}{$\begin{array}{l}\text { Invasive to adjacent } \\
\text { area }\end{array}$} & \\
\hline & $\overline{\mathrm{N}}$ & $\overline{\%}$ & $\overline{\mathrm{N}}$ & $\overline{\% \%}$ & $\overline{\mathrm{N}}$ & $\overline{\% \%}$ & $\overline{\mathrm{N}}$ & $\overline{\%}$ & $\overline{P P}$ \\
\hline peroxiredoxin 6-score & & & & & & & & & 0.008 \\
\hline Negative (0\%) & 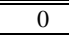 & 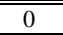 & 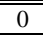 & 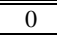 & 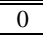 & 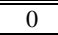 & $\overline{0}$ & 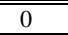 & \\
\hline Weak (1-25\%) & 1 & 7.1 & 1 & 7.7 & 0 & 0 & $\overline{0}$ & 0 & \\
\hline Moderate (26-50\%) & 8 & 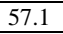 & 2 & 15.4 & 1 & 9.1 & 0 & 0 & \\
\hline $\begin{array}{l}\text { Strong }(51-75 \%) \\
\end{array}$ & $\overline{55}$ & 35.7 & 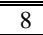 & 61.5 & $\overline{77}$ & 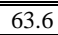 & $\overline{11}$ & 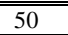 & \\
\hline "Very strong (76-100\%) & 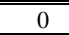 & $\overline{0}$ & 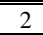 & 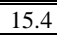 & 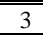 & 27.3 & $\overline{11}$ & 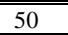 & \\
\hline Total & 14 & 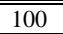 & 13 & 100 & 11 & 100 & 2 & 100 & \\
\hline Median & \multicolumn{2}{|c|}{ Moderate (26-50\%) } & \multicolumn{2}{|c|}{ Strong (51-75\%) } & \multicolumn{2}{|c|}{ Strong $(51-75 \%)$} & \multicolumn{2}{|c|}{$\begin{array}{c}\text { Very strong (76- } \\
100 \%)\end{array}$} & \\
\hline Mean Rank & \multicolumn{2}{|c|}{13.3} & \multicolumn{2}{|c|}{21.9} & \multicolumn{2}{|c|}{26.2} & \multicolumn{2}{|c|}{ 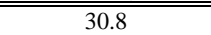 } & \\
\hline $\begin{array}{lll}r=0.543 & P<0.001\end{array}$ & & & & & & & & & \\
\hline
\end{tabular}

$*$ NS Non-significant relation ( $\mathrm{p}>0.05$ )

Table (8): The median value of selected marker scores by lymph Node involvement among OSCC cases group.

\begin{tabular}{|c|c|c|c|c|c|c|c|}
\hline & \multicolumn{6}{|c|}{ N (lymph Node involvement) } & \\
\hline & \multicolumn{2}{|c|}{ "No LN involvement } & \multicolumn{2}{|c|}{$\begin{array}{l}\text { N1 metastasis to single } \\
\text { ipsilateral LN } \leq 3 \mathrm{~cm}\end{array}$} & \multicolumn{2}{|c|}{$\begin{array}{l}\text { N2 metastasis to single } \\
\text { ipsilateral LN } 3-6 \mathrm{~cm}\end{array}$} & \\
\hline & $\overline{\mathrm{N}}$ & $\overline{\%}$ & $\mathrm{~N}$ & $\overline{\%}$ & $\overline{\mathrm{N}}$ & $\overline{\%}$ & $\overline{\bar{P}}$ \\
\hline peroxiredoxin 6-score & & & & & & & 0.012 \\
\hline Negative $(0 \%)$ & 0 & 0 & 0 & 0 & 0 & 0 & \\
\hline Weak (1-25\%) & 2 & 10.5 & 0 & 0 & 0 & 0 & \\
\hline Moderate (26-50\%) & 8 & 424.1 & 3 & \begin{tabular}{c|c|}
18.8 \\
\end{tabular} & 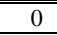 & 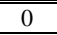 & \\
\hline Strong $(51-75 \%)$ & 8 & 42.1 & 10 & 62.5 & 3 & 60 & \\
\hline Very strong $(76-100 \%)$ & 1 & 5.3 & 3 & 18.8 & 2 & 40 & \\
\hline Total & 19 & 100 & 16 & 100 & 5 & 100 & \\
\hline Median & \multicolumn{2}{|c|}{ Moderate $(26-50 \%)$} & \multicolumn{2}{|c|}{ Strong $(51-75 \%)$} & \multicolumn{2}{|c|}{ Strong $(51-75 \%)$} & \\
\hline Mean Rank & \multicolumn{2}{|c|}{15.6} & \multicolumn{2}{|c|}{23.5} & \multicolumn{2}{|c|}{29.4} & \\
\hline $\mathrm{r}=0.474 \quad \mathrm{P}=0.002$ & & & & & & & \\
\hline
\end{tabular}

* NS Non-significant relation ( $>>0.05)$

Table (9): The median value of selected marker scores by tumour stage among OSCC cases group.

\begin{tabular}{|c|c|c|c|c|c|c|c|c|c|}
\hline & \multicolumn{8}{|c|}{ Tumor stage } & \multirow[b]{3}{*}{$\mathrm{P}$} \\
\hline & \multicolumn{2}{|c|}{ Stage-I } & \multicolumn{2}{|c|}{ Stage-II } & \multicolumn{2}{|c|}{ Stage-III } & \multicolumn{2}{|c|}{ Stage-IV } & \\
\hline & $\overline{\mathrm{N}}$ & $\%$ & $\mathrm{~N}$ & $\%$ & $\mathrm{~N}$ & $\%$ & $\mathrm{~N}$ & $\%$ & \\
\hline peroxiredoxin 6-score & & & & & & & & & 0.011 \\
\hline Negative (0\%) & 0 & 0 & 0 & 0 & 0 & $\overline{0}$ & 0 & 0 & \\
\hline Weak $(1-25 \%)$ & 1 & 9.1 & 0 & 0 & 1 & 5.9 & 0 & $\overline{0}$ & \\
\hline "Moderate (26-50\%) & $\overline{c 6}$ & 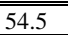 & 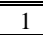 & 10.7 & 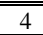 & 23.5 & $\overline{00}$ & 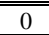 & \\
\hline $\begin{array}{l}\text { Strong }(51-75 \%) \\
\end{array}$ & 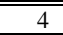 & 36.4 & 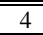 & 66.7 & 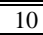 & 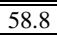 & 3 & 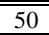 & \\
\hline Very strong $(76-100 \%)$ & 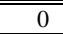 & $\overline{0}$ & 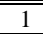 & 16.7 & 2 & 11.8 & 3 & $\overline{c 50}$ & \\
\hline Total & $\overline{11}$ & 100 & 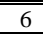 & 100 & 17 & 100 & $\overline{66}$ & 100 & \\
\hline Median & \multicolumn{2}{|c|}{ Moderate (26-50\%) } & \multicolumn{2}{|c|}{ Strong $(51-75 \%)$} & \multicolumn{2}{|c|}{ Strong $(51-75 \%)$} & \multicolumn{2}{|c|}{$\begin{array}{c}\text { Very strong (76- } \\
100 \%)\end{array}$} & \\
\hline Mean Rank & \multicolumn{2}{|c|}{13.2} & \multicolumn{2}{|c|}{23.6} & \multicolumn{2}{|c|}{20.5} & \multicolumn{2}{|c|}{30.8} & \\
\hline $\mathrm{r}=0.455 \quad \mathrm{P}=0.003$ & & & & & & & & & \\
\hline
\end{tabular}


Immunohistochemical Expression of Antioxidant Enzyme PRDX6 in Oral Squamous Cell Carcinoma In Relation to Clinicopathological Parameters Mustafa Gheny Taher

Table (10): The median value of selected marker scores by degree of tumour differentiation among cases with OSCC.

\begin{tabular}{|c|c|c|c|c|c|c|c|}
\hline & \multicolumn{6}{|c|}{ Degree of Differentiation (Grade) } & \\
\hline & \multicolumn{2}{|c|}{ Well Differentiated } & \multicolumn{2}{|c|}{ Moderate Differentiated } & \multicolumn{2}{|c|}{ Poor Differentiated } & \\
\hline & $\mathrm{N}$ & $\%$ & $\overline{\mathrm{N}}$ & $\%$ & $\mathrm{~N}$ & $\%$ & $\overline{\mathrm{P}}$ \\
\hline peroxiredoxin 6-score & & & & & & & $0.8[\mathrm{NS}]$ \\
\hline Negative $(0 \%)$ & 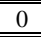 & 0 & 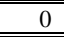 & 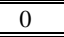 & 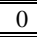 & 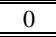 & \\
\hline Weak $(1-25 \%)$ & 1 & 4.5 & 0 & 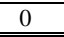 & 1 & 14.3 & \\
\hline Moderate (26-50\%) & 5 & 22.7 & 4 & 36.4 & 2 & 28.6 & \\
\hline Strong (51-75\%) & 13 & 59.1 & 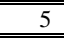 & 45.5 & 3 & 42.9 & \\
\hline Very strong (76-100\%) & 3 & 13.6 & 2 & 18.2 & 1 & 14.3 & \\
\hline Total & 22 & 100 & 11 & 100 & 7 & 100 & \\
\hline Median & \multicolumn{2}{|c|}{ Strong $(51-75 \%)$} & \multicolumn{2}{|c|}{ Strong $(51-75 \%)$} & \multicolumn{2}{|c|}{ Strong $(51-75 \%)$} & \\
\hline Mean Rank & \multicolumn{2}{|c|}{21.2} & \multicolumn{2}{|c|}{20.6} & \multicolumn{2}{|c|}{18.1} & \\
\hline$r=-0.091 \quad P=0.58[N S]$ & & & & & & & \\
\hline
\end{tabular}

Table (11): Area under ROC curve for selected marker when used as test to diagnose OSCC differentiating it from healthy controls.

\begin{tabular}{|l||c||c||c|c|}
\hline \multicolumn{1}{|c||}{} & \multicolumn{2}{c||}{ Percent positive cells } & \multicolumn{2}{c|}{ Marker score } \\
\hline \hline & AUROC & P & AUROC & P \\
\hline \hline peroxiredoxin 6 & 0.94 & $<0.001$ & 0.94 & $<0.001$ \\
\hline
\end{tabular}

Table (12): Validity parameters for selected markers when used as test to diagnose OSCC differentiating it from healthy controls.

\begin{tabular}{|c|c|c|c|c|c|c|}
\hline & & & & \multicolumn{2}{|c|}{$\begin{array}{l}\text { PPV at pretest } \\
\text { probability= }\end{array}$} & \multirow[t]{2}{*}{$\begin{array}{c}\text { NPV at pretest } \\
\text { probability }=10 \%\end{array}$} \\
\hline Positive if $\geq$ cut-off value & Sensitivity & Specificity & Accuracy & $50 \%$ & $90 \%$ & \\
\hline \multicolumn{7}{|l|}{ peroxiredoxin 6-Score } \\
\hline $\begin{array}{l}\text { Moderate }(26-50 \%) \text { (Highest } \\
\text { sensitivity and optimum cut-off } \\
\text { value) }\end{array}$ & 95.0 & 80.0 & 90.7 & 82.6 & 97.7 & 99.3 \\
\hline $\begin{array}{l}\text { Strong (51-75\%) (Highest } \\
\text { specificity) }\end{array}$ & $\overline{67.5}$ & 100.0 & $\overline{\overline{776.8}}$ & $\overline{100.0}$ & 100.0 & 96.5 \\
\hline
\end{tabular}

\section{Discussion}

The PRDX6 antioxidant enzyme is originate in a large range of classes and is important in the protection of cells against oxidants, in humans is encoded by the PRDX6 gene. It is a member of the PRDX family of antioxidant enzymes (Cong et al., 2013). (Neumann et al., 2007) had suggested that, since PRDXs are antioxidants, they support survival and tumor maintenance by protecting cells from oxidative stressinduced apoptosis. Also, it has been detected by Huang et al., 2011, that PRDX6 is associated with tumorigenesis in tongue SCC. In a similar manner, (Walsh et al.,
2009) reported that, the reduction of PRDX6 expression increased peroxide-induced cell death in liver cancer cells. In the present study, PRDX6 over expression in both, the mean cytoplasmic localization and the median score of PRDX6 were significantly higher in OSCC group than healthy control $\operatorname{group}(\mathrm{p}=0.001)$, confirming that the malignant cells, resist ROS during their high rate cellular proliferations, escaping apoptosis and allowing tumor growth. This findings agree with other researches done on OSCC cases (Huang et al., 2011; Huang et al., 2013;Lee et al., 2015) who reported that, 
the cancer cells showed increase the PRDX6 protein expression compared to the normal corresponding cells and found to be expressed at unusually high levels in several types of cancers including breast cancer (Chang et al., 2007). (Rolfs et al., 2013) found that, PRDX6 in keratinocytes of standard human skin and in the tumor cells of squamous cell carcinomas, representing a role of Prdx6 in human skin carcinogenesis. Whereas, (Young et al., 2015), found that there was no significant difference between OSCC and normal mucosa.

In the same context, concerning other malignant conditions, elevated levels of PRDX6 have been described in breast cancer (Thongwatchara et al., 2011), in malignant mesothelioma (Kinnula et al., 2002), in bladder cancer (Quan et al., 2006), in esophageal cancer (Zhang et al., 2011), in lung (Schremmer et al., 2007), ovarian (Pylväs et al., 2010) and pancreas (Park et al., 2011) cancer, in cancer of the gingivobuccal area (Shukla et al., 2009), and in lymphoma (Kuusisto et al., 2015).

Concerning age, in this study, most cases were in group (40-59) years of age and all of them were showed weak expression for PRDX6 and showed no important statistically significant difference in median score between the three age groups, also the expression was weak in both male and female and showed no statistically significant difference, also regarding site, the most affected region was present in the tongue with strong expression and showed no significant correlation with site distribution and to the best of knowledge, no such results in any study were reported.

This study showed that the the median score of PRDX6 was lowest among cases with T1 category and increase significantly with higher tumor size to reach its highest median score of very strong positivity among invading tumors to the adjacent areas ( $\mathrm{p}=0.008)$ also, this marker showed a statistically significant moderately strong positive linear correlation with tumor size $(r=0.543)$. Theses findings suggested that as the size of tumor increase, the antioxidant system increase it activity to resist the ROS that present during tumor cells proliferation (Lee et al., 2015). (Yi et al., 2013) on their study on esophageal SCC cases reported similar results, in contrast, (Young et al., 2015) and (Huang et al., 2013) reported that, there was no relation between tumor size and PROX6 in OSCC. Elevated expression levels of PRDX6 have been associated with a more invasive phenotype and metastatic potential of breast cancer (Chang et al., 2007; Arianna et al., 2017), and with a worse prognosis of clinically localized prostate cancer following radical prostatectomy (Raatikainen et al., 2015), such findings suggest that the PRDX6 is important during multi steps of tumor carcinogenesis including invasion (Lee et al., 2015) .

The observed difference in median score of PRDX6 between tumor lymph node involvement was statistically significant $(\mathrm{p}=0.012)$ with a statistically significant moderately strong positive linear correlation with tumor $\mathrm{N}$ stage $(\mathrm{r}=0.474)$. These findings 
agree with (Zhong et al., 2007) in their study on breast cancer. Such results suggested that, PROX6 can be used as predictable marker for lymph node metastasis of OSCC for early treatment and need more study confirmation. Other investigators as (Zhang et al., 2012;Huang et al., 2013; Yi et al., 2013) found opposite results with the present study findings in SCC cases. Recently in a study review explained the role of PRDX6 in several tumors and the role of PRDX6 in tumorigenesis, recurrence and invasion. (Nicolussi et al., 2017) they reported that, there was no relation with lymph node involvement. The invasion and metastasis promoting actions of PRDX6 has been found in lung cancer cells through activation of Akt via activation of phosphoinositiede 3-kinase (PI3K) and p38 kinase (Lee et al., 2009; Ho et al., 2010). The activity of PRDX6 contributes to the metastatic ability of lung cancer cells by stimulating invasion components including PI3K, Akt, and uPA (Ho et al., 2010). Overexpression of PRDX6 leads to a more invasive phenotype and metastatic potential in human breast cancer, through regulation of the levels of uPAR, Ets-1, MMP-9, RhoC and TIMP-2 expression (Chang et al., 2007). Regarding tumor stage, study findings showed that, the median score category of PRDX6 was lowest (moderate staining) among cases with tumors in the early stage (Stage-I) and progressively increase in magnitude of marker score to reach its highest value (very strong stain category score) among OSCC cases with highest tumor stage (Stage-IV) and also the observed difference in median score of PRDX6 between the four tumor stages was statistically significant. This marker showed a statistically significant moderately strong positive linear correlation with tumor $\mathrm{N}$ stage $(r=0.455)$, these results confirms that PRDX6 is an important antioxidant enzyme regulating the cellular redox state in malignant cells with potential effects on tumor growth by preventing apoptosis. So PRDX6 could be of a benefit in tumor staging of OSCC in addition to the traditional methods till more researches in the future will be conducted to confirm results.

Concerning the degree of tumour differentiation, a non significant correlation between expression of PRDX6 and degree of differentiation of OSCC cases was found in this study, this result agreed with (Huang et al., 2013) and such results were same as in pancreatic cancer (Lister et al., 2011), The exact molecular gadgets of how ROS and PRDX6 effect the carcinogenesis, however, remain sophisticated and unexplained to date, it is necessary to conduct further studies with a larger sample size to clarify the effect of this antioxidant in relation to cancer cells differentiation.

However, the molecular way between oxidant stress and the cellular redoxregulating enzymes in carcinomas have not yet been fully unstated, as well as the opposing reported significance of ROS and antioxidant systems within the carcinogenesis need more tests. 


\section{References}

[1]Salian Varsha, Dinakar Chethana, Shetty Pushparaja, Ajila Vidya . Etiological Trends in Oral Squamous Cell Carcinoma. A Retrospective Institutional Study. Cancer Translational Medicine journal 2016;2;2;3336.

[2]Molinolo A, Amornphimoltham $\mathrm{P}$, Squarize C, Castilho R, Patel V. Dysregulated molecular networks in head and neck carcinogenesis.Oral Oncol. 2009; 45: 324-334.

[3]Suni Ann Thomas and Sethupathy.S. Evaluaion of oxidative stress in patient with Oral Squamous cell carcinoma. Int J Pharm Bio Sci 2015 Jan; 6(1): (B) 289 - 293.

[4]Helmut Sies. Oxidative stress: a concept in redox biology and medicine. Science direct journals. Volume 4, April 2015, Pages 180-183.

[5]Halliwell B, Whiteman M (2004) Measuring reactive species and oxidative damage in vivo and in cell culture: how should you do it and what do the results mean? Br J Pharmacol 142:231-255.

[6]Azzi, K.J. Davies, F. Kelly Free radical biology - terminology and critical thinking FEBS Letters, 558 (1-3) (2004), pp. 3-6.

[7]A.L. Levonen, B.G. Hill, E. Kansanen, J. Zhan, V.M. Darley-Usmar Redox regulation of antioxidants, autophagy, and the response to stress: implications for electrophile therapeutics Free Radical Biology and Medicine, 71 (2014), pp. 196-207.

[8]Cong-Fa Huang, Lu Zhang, Si-Rui Ma, Zhi-Li Zhao, Wei-Ming Wang, Ke-Fei He, Yi-Fang Zhao, Wen-Feng Zhang, Bing Liu,
Zhi-Jun Sun PLoS One. 2013; PMC3873935, 8(12): e83479. Published online 2013 December 27.

[9]LEE E-Y, KANG J-Y, KIM K-W. Expression of cyclooxygenase-2, peroxiredoxin I, peroxiredoxin 6 and nuclear factor- $\mathrm{\kappa B}$ in oral squamous cell carcinoma. Oncology Letters. 2015;10(5):3129-3136. [10] Kinnula VL, Lehtonen S, Sormunen R, Kaarteenaho-Wiik R, Kang SW, Rhee SG, Soini Y. Overexpression of peroxiredoxins I, II, III, V, and VI in malignant mesothelioma. J Pathol. 2002; 196:316-323.

[11]Neumann CA, Fang Q (2007) Are peroxiredoxins tumor suppressors? Curr Opin Pharmacol 7: 375-380.

[12]Walsh B, Pearl A, Suchy S, Tartaglio J, Visco K, et al. (2009) Overexpression of Prdx6 and resistance to peroxide-induced death in Hepa1-6 cells: Prdx suppression increases apoptosis. Redox Rep 14: 275-284. [13]Ho JN, Lee SB, Lee SS, Yoon SH, Kang GY, et al. (2010) Phospholipase A2 activity of peroxiredoxin 6 promotes invasion and metastasis of lung cancer cells. Mol Cancer Ther 9: 825-832.

[14]Lee SB, Ho JN, Yoon SH, Kang GY, Hwang SG, et al. (2009) Peroxiredoxin 6 promotes lung cancer cell invasion by inducing urokinase-type plasminogen activator via p38 kinase, phosphoinositide 3kinase, and Akt. Mol Cells 28: 583-588.

[15]Schremmer B, Manevich Y, Feinstein SI, Fisher AB (2007) Peroxiredoxins in the lung with emphasis on peroxiredoxin VI. Subcell Biochem 44: 317-344. 
Immunohistochemical Expression of Antioxidant Enzyme PRDX6 in Oral Squamous Cell Carcinoma In Relation to Clinicopathological Parameters

Mustafa Gheny Taher

[16]Jhang Ho Pak, Won Ho Choi, Hye Mi Lee, Won-Duk Joo, Jong-Hyeok Kim, Young-Tak Kim, Yong-Man Kim \& JooHyun Nam.Peroxiredoxin 6 Overexpression Attenuates Cisplatin-Induced Apoptosis in Human Ovarian Cancer Cells.Journal Cancer Investigation Volume 29, 2011 - Issue 1. [17]Rolfs F1, Huber M, Gruber F, Böhm F, Pfister HJ, Bochkov VN, Tschachler E, Dummer R, Hohl D, Schäfer M, Werner S. Dual role of the antioxidant enzyme peroxiredoxin 6 in skin carcinogenesis..Cancer Res. 2013 Jun 1;73(11):3460-9.

[18]Sharapov MG, Gudkov SV, Gordeeva AE, Karp OE, Ivanov VE, Shelkovskaya OV, Bruskov VI, Novoselov VI6, Fesenko EE.Peroxiredoxin 6 is a natural radioprotector.Dokl Biochem Biophys. 2016 Mar;467(1):110-2.

[19]C-F Huang, Z-J Sun, Y-F Zhao, X-M Chen, J Jia, W-F Zhang; Increased expression of peroxiredoxin 6 and cyclophilin A in squamous cell carcinoma of the tongue, Oral Diseases (2011) 17, 328334

[20]Xin-Zhong Chang, Da-Qiang Li, YiFeng Hou, Jiong Wu, Jin-Song Lu, GenHong Di, Wei Jin, Zhou-Luo Ou, Zhen-Zhou Shen, and Zhi-Ming. Identification of the functional role of peroxiredoxin 6 in the progression of breast cancer. Breast Cancer Res. 2007; 9(6): R76.

[21] EUN-YOUNG LEE, JI-YEON KANG and KYOUNG-WON KIM. Expression of cyclooxygenase-2, peroxiredoxin I, peroxiredoxin 6 and nuclear factor- $\mathrm{\kappa B}$ in oral squamous cell carcinoma. ONCOLOGY LETTERS 10: 3129-3136, 2015.

[22]Thongwatchara P, Promwikorn W, Srisomsap C, Chokchaichamnankit D, Boonyaphiphat P, Thongsuksai P. Differential protein expression in primary breast cancer and matched axillary node metastasis. Oncol Rep. 2011; 26:185-191. [23]Quan C, Cha EJ, Lee HL, Han KH, Lee KM, Kim WJ. Enhanced expression of peroxiredoxin I and VI correlates with development, recurrence and progression of human bladder cancer. J Urol. 2006; 175:1512-1516.

[24]Zhang J, Wang K, Zhang J, Liu SS, Dai $\mathrm{L}$, Zhang JY. Using proteomic approach to identify tumor-associated proteins as biomarkers in human esophageal squamous cell carcinoma. J Proteome Res. 2011; 10:2863-2872.

[25]Pylväs M, Puistola U, Kauppila S, Soini Y, Karihtala P. Oxidative stress-induced antioxidant enzyme expression is an early phenomenon in ovarian carcinogenesis. Eur J Cancer. 2010; 46:1661-1667.

[26]Park JY, Kim SA, Chung JW, Bang S, Park SW, Paik YK, Song SY. Proteomic analysis of pancreatic juice for the identification of biomarkers of pancreatic cancer. J Cancer Res Clin Oncol. 2011; 137:1229-1238.

[27]Shukla S, Pranay A, D'Cruz AK, Chaturvedi P, Kane SV, Zingde SM (2009). Immunoproteomics reveals that cancer of the tongue and the gingivobuccal complex exhibit differential autoantibody response. Cancer Biomark 5: 127-135. 
[28]Kuusisto ME, Haapasaari KM, Turpeenniemi-Hujanen T, Jantunen E, Soini Y, Peroja P, Bloigu R, Karihtala P, Kuittinen O. High intensity of cytoplasmic peroxiredoxin VI expression is associated with adverse outcome in diffuse large B-cell lymphoma independently of international prognostic index. J Clin Pathol. 2015; 68:552-556.

[29]Yi Li, Hui Guo, Danfeng Dong, Huili $\mathrm{Wu}$, Enxiao Li.Expression and prognostic relevance of Cyclophilin A and matrix metalloproteinase 9 in esophageal squamous cell carcinoma . Diagn Pathol. 2013; 8: 207. [30]Arianna Nicolussi,1 Sonia D'Inzeo,1 Carlo Capalbo,2 Giuseppe Giannini,2 and Anna Coppa1; he role of peroxiredoxins in cancer;Mol Clin Oncol. 2017 Feb; 6(2): 139153.

[31]Raatikainen S, Aaaltomaa S, Kärjä V, Soini Y. Increased peroxiredoxin 6 expression predicts biochemical recurrence in prostate cancer patients after radical prostatectomy. Anticancer Res. 2015; 35:6465-6470.
[32]Zhang X, Su Y, Zhang M \& Sun Z 2012 Opposite effects of arsenic trioxide on the Nrf2 pathway in oral squamous cell carcinoma in vitro and in vivo. Cancer Letters 318 93-98.

[33]Arianna Nicolussi,1 Sonia D'Inzeo,1 Carlo Capalbo,2 Giuseppe Giannini,2 and Anna Coppa1; he role of peroxiredoxins in cancer;Mol Clin Oncol. 2017 Feb; 6(2): 139153.

[34]Lee SB, Ho JN, Yoon SH, Kang GY, Hwang SG, et al. (2009) Peroxiredoxin 6 promotes lung cancer cell invasion by inducing urokinase-type plasminogen activator via p38 kinase, phosphoinositide 3kinase, and Akt. Mol Cells 28: 583-588. [35]Lister A, Nedjadi T, Kitteringham NR, Campbell F, Costello E, et al. (2011) Nrf2 is overexpressed in pancreatic cancer: implications for cell proliferation and therapy. Mol Cancer 10: 37. 